\title{
Multiple-Input Single-Output Control for Extending the Steady-State Operating Range-Use of Controllers with Different Setpoints
}

\author{
Adriana Reyes-Lúa ${ }^{+}(\mathbb{D})$ and Sigurd Skogestad $*,+(\mathbb{D}$ \\ Department of Chemical Engineering, Norwegian University of Science and Technology (NTNU), \\ Sem Sælands vei 4, 7491 Trondheim, Norway; adriana.r.lua@sintef.no \\ * Correspondence: sigurd.skogestad@ntnu.no \\ + These authors contributed equally to this work.
}

Received: 15 November 2019; Accepted: 5 December 2019; Published: 10 December 2019

check for updates

\begin{abstract}
This paper deals with a case when multiple inputs are needed to cover the steady-state operating range. The most common implementation is to use split range control with a single controller. However, this approach has some limitations. In this paper, we use multiple controllers with different setpoints and demonstrate that this structure can be optimal in some cases when the cost of the input can be traded off against the penalty of deviating from the desired setpoint. We describe a procedure to find the optimal setpoint deviations. We illustrate our procedure in a case in which three inputs (cooling and two sources of heating) are used to control the temperature of a room with a PID-based control structure and without the need of online optimization.
\end{abstract}

Keywords: control structure; optimal operation; PID; split range control; valve position control; parallel control

\section{Introduction}

The use of more than one input for one output to extend the steady-state range of the output has been a common practice for more than 75 years (e.g., References [1-3]). Note that in this paper, we use input $(u)$ as a synonym of manipulated variable (MV) and output $(y)$ as a synonym of controlled variable (CV). Split range control is the classical control structure commonly used for this. However, using a single controller has some limitations with respect to tuning. For example, for split range with PI control, the integral times must be the same for all inputs.

An alternative to extend the steady-state range of the output is to use one controller for each input with independent tunings and different setpoints. This structure is often regarded as "sub-optimal" because the setpoints must be different to avoid undesired switching of the controllers [4]. In this paper, we argue that having different setpoints can be optimal in some cases, because it allows us to consider the trade-off between the cost of using the input against the cost of deviating from the desired setpoint. For example, for room temperature control we may use different setpoints in the winter than in the summer to save on heating and cooling, respectively.

This paper is organized as follows. In Section 2 we describe the classical control structures used to extend the steady-state range and maintain control of the output when there is more than one available input. In Section 3 we introduce our proposed procedure to obtain optimal setpoints. In Section 4 we implement our proposed procedure in a case study in which we find optimal setpoints for controlling the temperature of a room with three inputs. In Section 5, we discuss the validity and the applicability of our method with objective functions different than those in Sections 3 and 4. We give some final remarks in Section 6. 


\section{Classical Advanced Control Structures for More than One Input for One Output}

When we need more than one input $\left(u_{i}\right.$, manipulated variable, MV) to cover the whole steady-state range for one output $(y$, controlled variable, CV), we can use three alternative classical control structures [5]:

1. Split range control (Figures 1 and 2)

2. Input (valve) position control (Figure 3)

3. One controller for each input, each with a different set point for the output (Figure 4)

Split range control has been in use for more than 75 years $[1,2]$ and it is still commonly implemented in industry [6]. Figure 1 shows the block diagram of a split range controller (SRC) with two inputs $\left(u_{1}\right.$ and $\left.u_{2}\right)$ for one output $(y)$. Here, there is a common controller (C) that produces an internal signal in deviation variables $(v)$ that is the input to the split range (SR) block, which calculates the values for $u_{i}$ (in physical variables) [7].

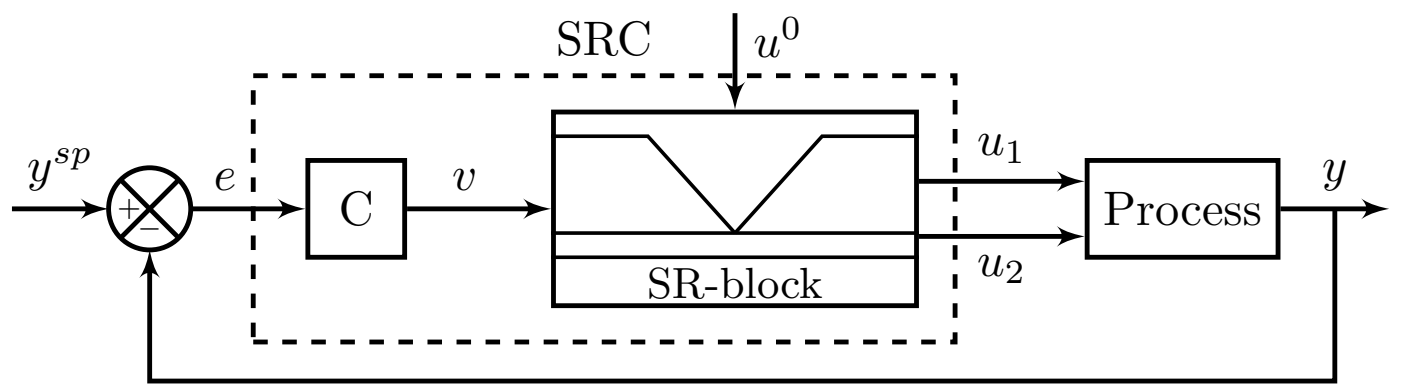

Figure 1. Alternative 1 for extending the steady-state range with MISO control: Classical split range control (SRC) for the case with two inputs and one output. Another typical split range block (SR-block) is shown in Figure 2.

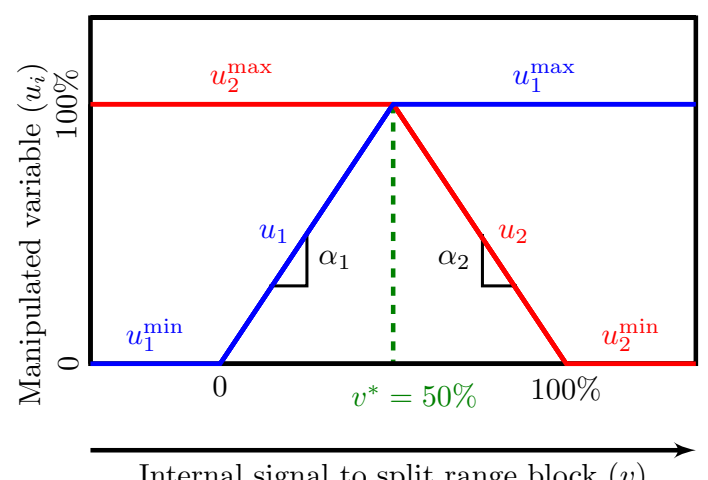

(a)

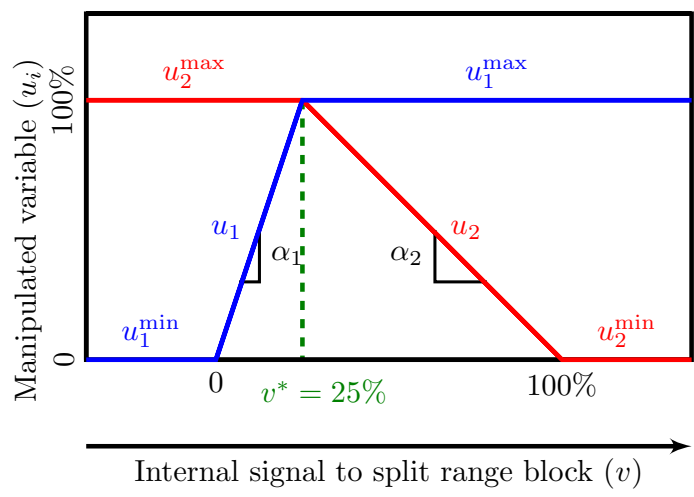

(b)

Figure 2. Split range block (SR-block) in Figure 1 for a case in which the two inputs have different signs for the steady-state gain. (a) Typical case, with $v^{*}=50 \%$. (b) More general case, with $v^{*} \neq 50 \%$.

Figure 2 shows a typical split range block. When the internal control signal $(v)$ is below the split value $\left(v^{*}\right), u_{1}$ is used to control $y$, while $u_{2}$ is saturated; whereas when $v$ is above $v^{*}, u_{1}$ is used to control $y$. The split point $\left(v^{*}\right)$ or, equivalently, the corresponding slopes $\left(\alpha_{i}\right)$ in Figure 2 , can be used as degrees of freedom to counteract the differences in the effects of the various inputs $\left(u_{i}\right)$. The approach introduced in Reference [7] considers not only the static effect but also the dynamics. Nevertheless, there are limitations in terms of tuning, as only the controller gains can be adjusted using the slopes; for example, the integral time needs to be the same for all inputs.

Alternative 2, shown in Figure 3, is input (valve) position control [8,9]. Valve position control is often used to improve the dynamic performance by allowing $u_{1}$ to take care of the fast control and $u_{2}$ 
of the long-term control. However, if implemented as shown in Figure 3, it extends the steady-state range. In this scheme, the primary input $\left(u_{1}\right)$ always controls the output $(y)$. If $u_{1}$ approaches its limit $\left(u_{1}^{\text {lim }}\right.$, either $u_{1}^{\min }$ or $u_{1}^{\max }$ ), then $u_{2}$ is used to control $u_{1}$ at a setpoint $u_{1}^{s p}=u_{1}^{\text {lim }}+\Delta u_{1}$, preventing $u_{1}$ from saturating. Note than $u_{2}$ is only controlling $u_{1}$ when needed, so it will normally be kept at its desired (nominal) value, $u_{2}^{0}$. We need to have a back-off from the limit $\left(u_{1}^{\text {lim }}\right)$ to ensure that $u_{1}$ always has some range to control $y$. Thus, $\Delta u_{1} \neq 0$ and one cannot utilize the full steady-state range of $u_{1}$ with this scheme.

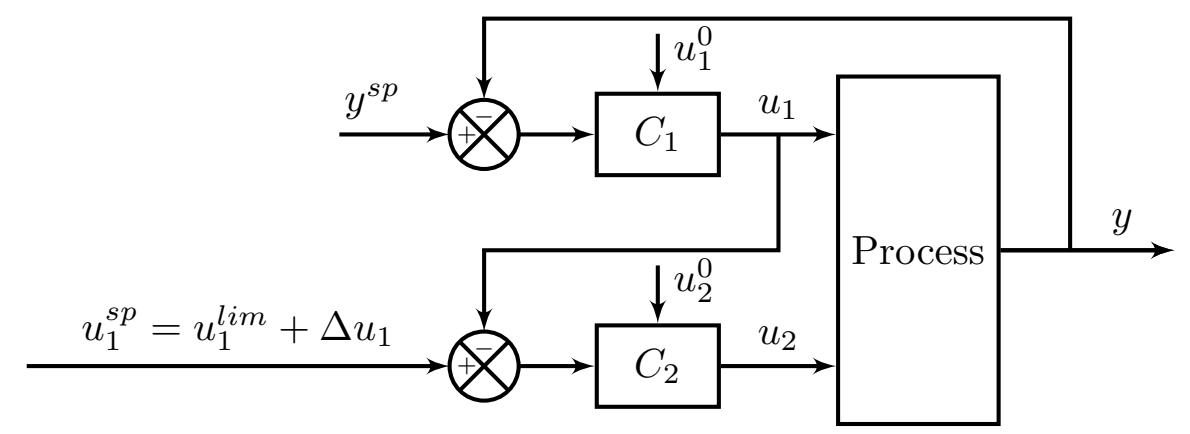

Figure 3. Alternative 2 for extending the steady-state range with MISO control: Input (valve) position control where input $u_{2}$ is used when $u_{1}$ reaches its limit.

Finally, Figure 4 shows alternative 3, studied in this paper, with one controller for each input. In Figure 4, the setpoint for the controller using $u_{1}\left(C_{1}\right)$ is $y^{s p, 1}$ and the setpoint for the controller using $u_{2}\left(C_{2}\right)$ is $y^{s p, 1}+\Delta y^{s p}$. Here, $\Delta y^{s p}$ should be large enough to guarantee that only one controller is active at a given time, while the other inputs are at their limits [10]. Compared to the split range control structure in Figure 1, the structure in Figure 4 has the advantage that the controllers can be tuned independently. However, it is normally considered a disadvantage that the setpoints must be different but in the next section we argue that there are cases where this is actually an advantage.

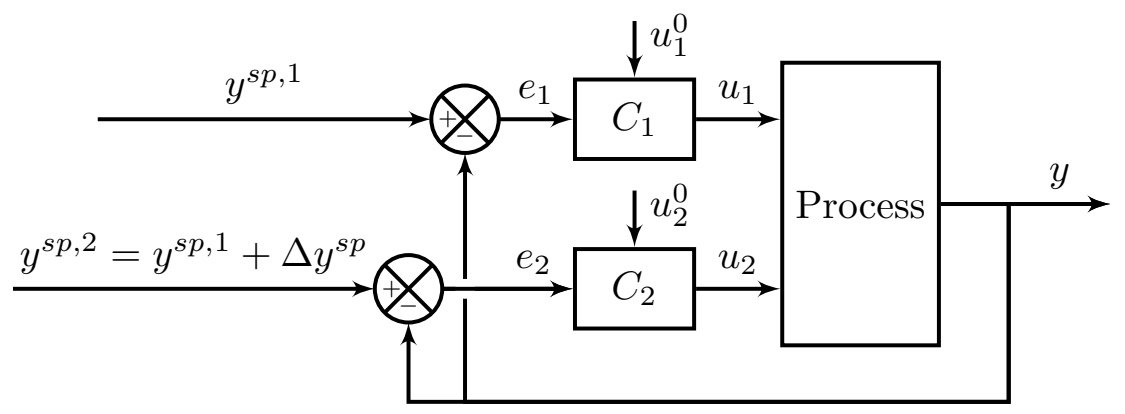

Figure 4. Alternative 3 for extending the steady-state range with MISO control: Two controllers with different setpoints for the same output. This is the control structure studied in this paper.

In Figures 1, 3 and 4 we show the case with two inputs $\left(u_{1}\right.$ and $\left.u_{2}\right)$ but all three alternatives are easily generalized to any number of inputs. For all three alternatives, the idea is that only one input $\left(u_{k}\right)$ is controlling the output $(y)$ at a time. In Figure 1 this is achieved by the split range block. In Figure 3, input $u_{2}$ is only used when $u_{1}$ reaches its limit. In Figure 4 , this is achieved by having different setpoints with sufficiently large $\Delta y^{s p}$.

In this paper, we study in detail one controller for each input (Figure 4) and we compare this structure with split range control (Figure 1).

\section{Optimal Setpoint for Each Input}

In this section we consider the cases when there is a trade-off between the cost of input usage $\left(u_{i}\right)$ and the cost of deviation from the setpoint $\left(\Delta y^{s p}\right)$. 
As only one input is being used at a time, the cost function (economic objective function) can be written as

$$
J\left(u_{k}, \Delta y^{s p}\right),
$$

where $u_{k}$ is the input usage for the active input and $\Delta y^{s p}$ is the deviation from the desired setpoint $\left(y-y^{s p}\right)$. We assume here that the cost is linear in $u$ and we assume a quadratic penalty for the setpoint deviation. Then the cost function, which we want to minimize, becomes

$$
J=p_{u_{k}} u_{k}+p_{y}\left(y-y^{s p}\right)^{2}+c,
$$

where $p_{u_{k}}$ is the price for input usage, $p_{y}$ represents the price for deviating from the desired setpoint and $c$ represents the cost related to keeping the other inputs $\left(u_{i}, \forall i, i \neq k\right)$ at their maximum or minimum values (not used to control y).

The output $(y)$ is a function of the inputs $(u)$. We consider the steady-state when we have

$$
y=f(u) .
$$

If we consider the case where the relationship in Equation (3) is linear for all inputs $\left(u_{i}\right)$, we then have that all inputs can be written as a linear function of $y$. Thus,

$$
u_{i}=k_{i} y+u_{i, 0} .
$$

The cost, when using $u_{k}$ as the input, then becomes

$$
J=p_{u_{k}} k_{k} y+p_{y}\left(y-y^{s p}\right)^{2}+c_{k}+p_{u_{k}} u_{k, 0} .
$$

The optimal value of the input $\left(y^{*}\right)$, which minimizes the cost $J$ when using input $u_{k}$ is then given by

$$
\frac{d J}{d y}=0 \Rightarrow p_{u_{k}} k+2 p_{y}\left(y^{*}-y^{s p}\right)=0 .
$$

We find that the optimal setpoint deviation is

$$
\Delta y^{s p^{*}}=y^{*}-y^{s p}=-\frac{p_{u_{k}} k}{2 p_{y}} .
$$

Thus, in this case, it is optimal with a constant setpoint deviation, independent of any other disturbances. Of course, this will not be the case if we have a different cost function than Equation (2) or a model which is not linear like Equation (4).

An example of a problem that satisfies our assumptions of a linear model is the heating or cooling of a room. The energy balance is

$$
0=\alpha\left(T^{a m b}-T\right)+Q_{h}-Q_{c} .
$$

$y=T$ is the room temperature, $Q_{h}$ represents the net heating and $Q_{c}$ the net cooling. The term $\alpha\left(T^{a m b}-T\right)$ represents the net heat loss to the environment. Equation (8) can be written on the form in Equation (4) with $y=T, u_{0}=\alpha T^{a m b}$ and

$$
k=\left\{\begin{array}{rlll}
\alpha & \text { if } & u_{k}=Q_{h} & \text { (heating) } \\
-\alpha & \text { if } & u_{k}=Q_{c} & \text { (cooling) }
\end{array} .\right.
$$

In general, the optimal setpoint deviation will not be independent of disturbances, as it is in Equation (7). It only holds when Equations (3) and (4) are valid. 


\section{Case Study}

Here we will analyze temperature control for the room in Figure 5, which can be described by Equation (8). The detailed model and the parameters are found in Appendix A. The desired (ideal) temperature in the room is $T^{s p}=21^{\circ} \mathrm{C}$. The main disturbance is ambient temperature $\left(d=T^{a m b}\right)$ and there are three available manipulated variables $\left(u_{i}\right)$ :

- $u_{1}=Q_{A C}:$ cooling using air conditioning

- $u_{2}=Q_{H W}$ : hot water, through floor heating $\left(Q_{F L}\right)$

- $u_{3}=Q_{E H}$ : electrical heating.

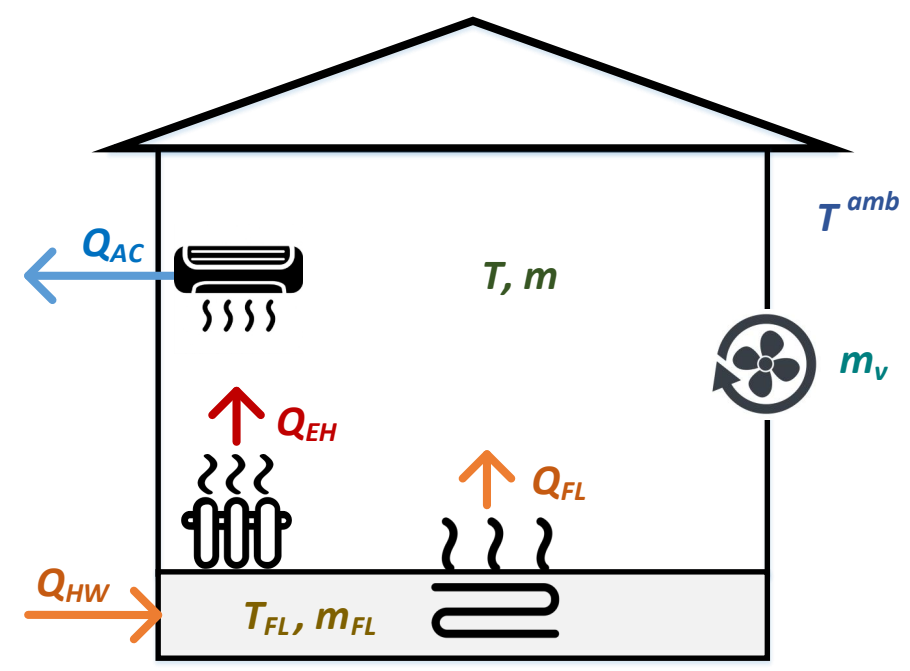

Figure 5. Room with three independent inputs $\left(u_{i}=Q_{i}\right)$ for controlling temperature $(y=T)$.

We select the nominal operating point as $T=T^{a m b}=21^{\circ} \mathrm{C}$. We use air conditioning $\left(u_{1}=Q_{A C}\right)$ to lower the temperature when $T^{a m b}>21^{\circ} \mathrm{C}$. When $T^{a m b}<21^{\circ} \mathrm{C}$ and the room requires heating, we first use hot water $\left(u_{2}=Q_{H W}\right)$ and when it reaches its maximum, we use electric heating $\left(u_{3}=Q_{E H}\right)$. Therefore:

$$
\begin{aligned}
& u=\left[Q_{A C}, Q_{H W}, Q_{E H}\right]^{\top} \\
& d=T^{a m b} \\
& y=T .
\end{aligned}
$$

The nominal values and ranges for the inputs $\left(u_{k}\right)$ are shown in Table 1.

Table 1. Ranges for available inputs $\left(u_{k}\right)$.

\begin{tabular}{clcccc}
\hline Input $\left(\boldsymbol{u}_{\boldsymbol{k}}\right)$ & Description & Nominal & Min & Max & Units \\
\hline$u_{1}=Q_{A C}$ & air conditioning & 0 & 0 & 4.5 & $\mathrm{~kW}$ \\
$u_{2}=Q_{H W}$ & heating water & 0 & 0 & 3.0 & $\mathrm{~kW}$ \\
$u_{3}=Q_{E H}$ & electrical heating & 0 & 0 & 4.0 & $\mathrm{~kW}$ \\
\hline
\end{tabular}

\subsection{Optimal Operation for Temperature Control}

We define a scalar cost function which takes into account the cost of energy as well as a quadratic penalty cost for deviating from the temperature setpoint. 


$$
J=\underbrace{\text { Jenergy }}_{\underbrace{p_{A C} Q_{A C}}_{p_{1} u_{1}}+\underbrace{p_{H W} Q_{H W}}_{p_{2} u_{2}}+\underbrace{p_{E H} Q_{E H}}_{p_{3} u_{3}}}+\underbrace{p_{T}\left(T-T^{s p}\right)^{2}}_{p_{y}\left(y-y^{s p}\right)^{2}}[\$ / s],
$$

where $p_{E H}, p_{H W}$ and $p_{A C}$ are the energy prices for electric heating $\left(Q_{E H}\right)$, heating water $\left(Q_{H W}\right)$ and air conditioning $\left(Q_{A C}\right)$. $p_{T}$ is a "comfort" penalty for the deviation of the actual room temperature ( $T$ ) from the desired room temperature $\left(T^{s p}\right)$. The values for these prices are in Table 2. Note that Equation (10) has the same form as Equation (5) when only one input is active.

Table 2. Parameters for cost function for optimization.

\begin{tabular}{clc}
\hline Parameter & Description & Price \\
\hline$p_{E H}$ & price for electrical heating & $1.20 \$ / \mathrm{kWh}$ \\
$p_{H W}$ & price for heating water & $0.80 \$ / \mathrm{kWh}$ \\
$p_{A C}$ & price for air conditioning & $0.40 \$ / \mathrm{kWh}$ \\
$p_{T}$ & comfort penalty & $0.24 \$ /{ }^{\circ} \mathrm{C}^{2} \mathrm{~h}$ \\
\hline
\end{tabular}

With the prices in Table 2 one hour of use of maximum heating water $(3 \mathrm{~kW})$ and maximum electricity $(4 \mathrm{~kW})$ costs

$$
(1.2 \$ / \mathrm{kWh})(4 \mathrm{kWh})+(0.8 \$ / \mathrm{kW})(3 \mathrm{kWh})=7.2 \$,
$$

whereas one hour with a $1{ }^{\circ} \mathrm{C}$ deviation costs $0.24 \$$.

\subsection{Optimal Setpoints for Room Temperature}

We want to find the optimum steady-state value for the room temperature, considering economics and deviation from the desired room temperature (Equation (10)). To this end, we analyze the effect of varying the temperature setpoint when we use different inputs on the economic optimum of the system. At steady-state, the energy balance for the room becomes:

$$
0=\alpha\left(T^{a m b}-T\right)+Q_{H W}+Q_{E H}-Q_{A C}[\mathrm{~kW}] .
$$

See also Equation (8).

For illustration purposes, we consider the case when $Q_{A C}$ is the active input, while $Q_{H W}=Q_{H W}^{\min }$ and $Q_{E H}=Q_{E H}^{\min }$. Then, Equation (11) becomes:

$$
0=\alpha\left(T^{a m b}-T\right)+Q_{H W}^{\min }+Q_{E H}^{\min }-Q_{A C} .
$$

Note that with $Q_{H W}^{m i n}=0$ and $Q_{E H}^{m i n}=0$, the steady-state room temperature is

$$
T=T^{a m b}-\frac{Q_{A C}}{\alpha} .
$$

Considering Equations (10) and (12).

$$
\left.\frac{d J}{d T}\right|_{Q_{E H}, Q_{H W}}=-\alpha p_{A C}+2 p_{T}\left(T-T^{s p}\right) .
$$

We find the optimal temperature from Equation (14), $\left.\frac{d J}{d T}\right|_{Q_{E H}, Q_{H W}}=0$ and we choose this as our setpoint when we use air conditioning (AC).

$$
T_{A C}^{s p}=T^{s p}+\frac{\alpha p_{A C}}{2 p_{T}} .
$$


This same analysis is valid for the case in which $Q_{H W}$ or $Q_{E H}$ are the active inputs. This result corresponds to Equations (7) and (9). Thus, the optimal setpoint deviations, when using only one input at a time are:

$$
\begin{gathered}
\Delta y^{s p, 1}=T_{A C}^{s p}-T^{s p}=+\frac{\alpha p_{a c}}{2 p_{T}} \\
\Delta y^{s p, 2}=T_{H W}^{s p}-T^{s p}=-\frac{\alpha p_{h w}}{2 p_{T}} \\
\Delta y^{s p, 3}=T_{E H}^{s p}-T^{s p}=-\frac{\alpha p_{e l}}{2 p_{T}} .
\end{gathered}
$$

With $p_{T}>0$, the deviation of $T$ from $T^{s p}$ is always penalized. If the comfort penalty $\left(p_{T}\right)$ is very high, $T_{i}^{s p} \approx T^{s p}$ in equations, (16a)-(16c).

For example, consider $i=1$ (AC). Then, from Equation (16a) and with the prices from Table 2 and data from Table A1

$$
\Delta y^{s p, 1}=\Delta T_{A C}^{s p}=\frac{400 \frac{\mathrm{W}}{{ }^{\circ} \mathrm{C}} 0.04 \frac{\$}{\mathrm{kWh}}}{(2)\left(0.24 \frac{\$}{{ }^{\circ} \mathrm{C}^{2} \mathrm{~h}}\right)}=0.33{ }^{\circ} \mathrm{C} .
$$

The results for all the inputs are in Table 3. The results are also shown graphically in Figure 6 as a function of $p_{i} / p_{T}$ for the case with $\alpha=400 \mathrm{~W} /{ }^{\circ} \mathrm{C}$.

Table 3. Optimal deviations for the three available inputs.

\begin{tabular}{cc}
\hline Input & $\boldsymbol{\Delta} \boldsymbol{y}^{\text {sp, } \boldsymbol{i}}\left[{ }^{\circ} \mathbf{C}\right]$ \\
\hline$u_{1}=Q_{A C}$ & +0.33 \\
$u_{2}=Q_{H W}$ & -0.67 \\
$u_{3}=Q_{E H}$ & -1.00 \\
\hline
\end{tabular}

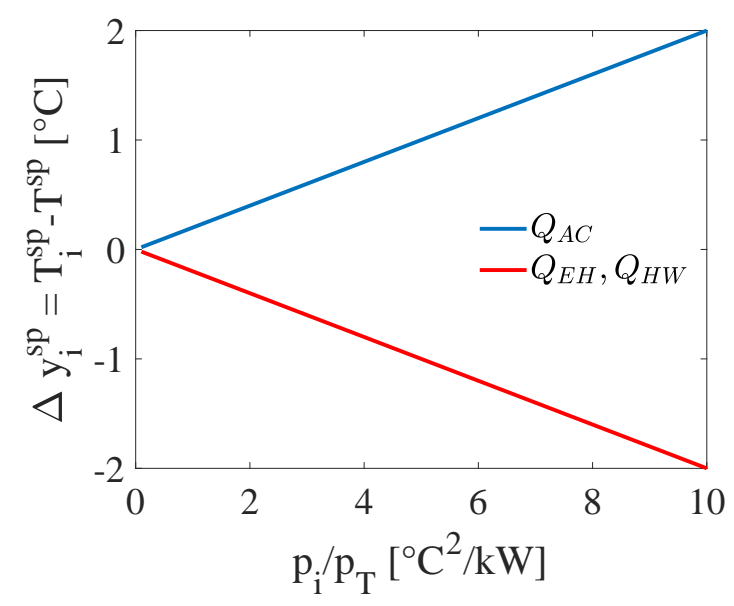

Figure 6. Effect of comfort penalty $\left(p_{T}\right)$ and input usage $\left(p_{i}\right)$ on optimal setpoint deviation $\left(\Delta y^{s p, i}\right)$.

\subsection{Three Controllers with Different Setpoints}

We can implement the results in Section 4.2 using a controller for each input each with a different setpoint, as shown in Figure 7, with $y=T$ and $u_{i}=Q_{i}$. The tuning procedure for the PI controllers is described in Appendix B.

Figure 8 shows the simulation results using large steps in $d=T^{a m b}$ to show the performance of the control structure in the whole range. All controllers have anti-windup (clamping) implemented. We use the optimal setpoints in Table 3. 


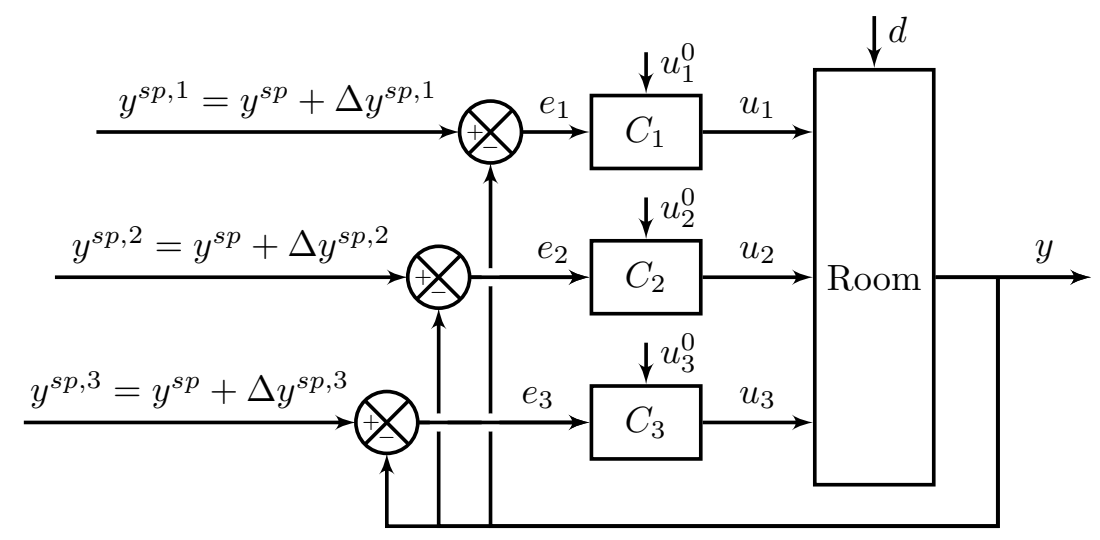

Figure 7. Block diagram for three controllers with different setpoints, one for each input; $y=T$, $d=T^{a m b}$ and $u_{i}=Q_{i}$, where $1=\mathrm{AC}, 2=\mathrm{HW}, 3=\mathrm{EH}$.

The simulation starts at the nominal point, with $T=T^{a m b}=21^{\circ} \mathrm{C}$. At $t=0.5 \mathrm{~h}, T^{a m b}$ increases by $+10^{\circ} \mathrm{C}$ and we need air conditioning $\left(Q_{A C}\right)$ to cool down the room. We observe that $T$ reaches $T_{A C}^{s p}=21.33{ }^{\circ} \mathrm{C}$ at steady-state. At $t=3.5 \mathrm{~h}, T^{a m b}$ is decreased by $-5{ }^{\circ} \mathrm{C}$ to $20^{\circ} \mathrm{C}$ and we keep using $Q_{A C}$ as input, reaching again $T_{A C}^{s p}=21.33^{\circ} \mathrm{C}$ at steady-state. Then, at $t=7 \mathrm{~h}, T^{a m b}$ is decreased by $-8{ }^{\circ} \mathrm{C}$ to $18{ }^{\circ} \mathrm{C}$ and we now use $Q_{H W}$ as input and we reach $T=T_{H W}^{s p}=20.33^{\circ} \mathrm{C}$ at steady-state. At $t=10 \mathrm{~h}, T^{a m b}$ is further decreased by $-13^{\circ} \mathrm{C}$ to $5^{\circ} \mathrm{C}$. $Q_{H W}$ reaches $Q_{H W}^{m a x}$, such that $Q_{E H}$ becomes the active input and $T=T_{E H}^{s p}=20.33^{\circ} \mathrm{C}$ at steady-state.
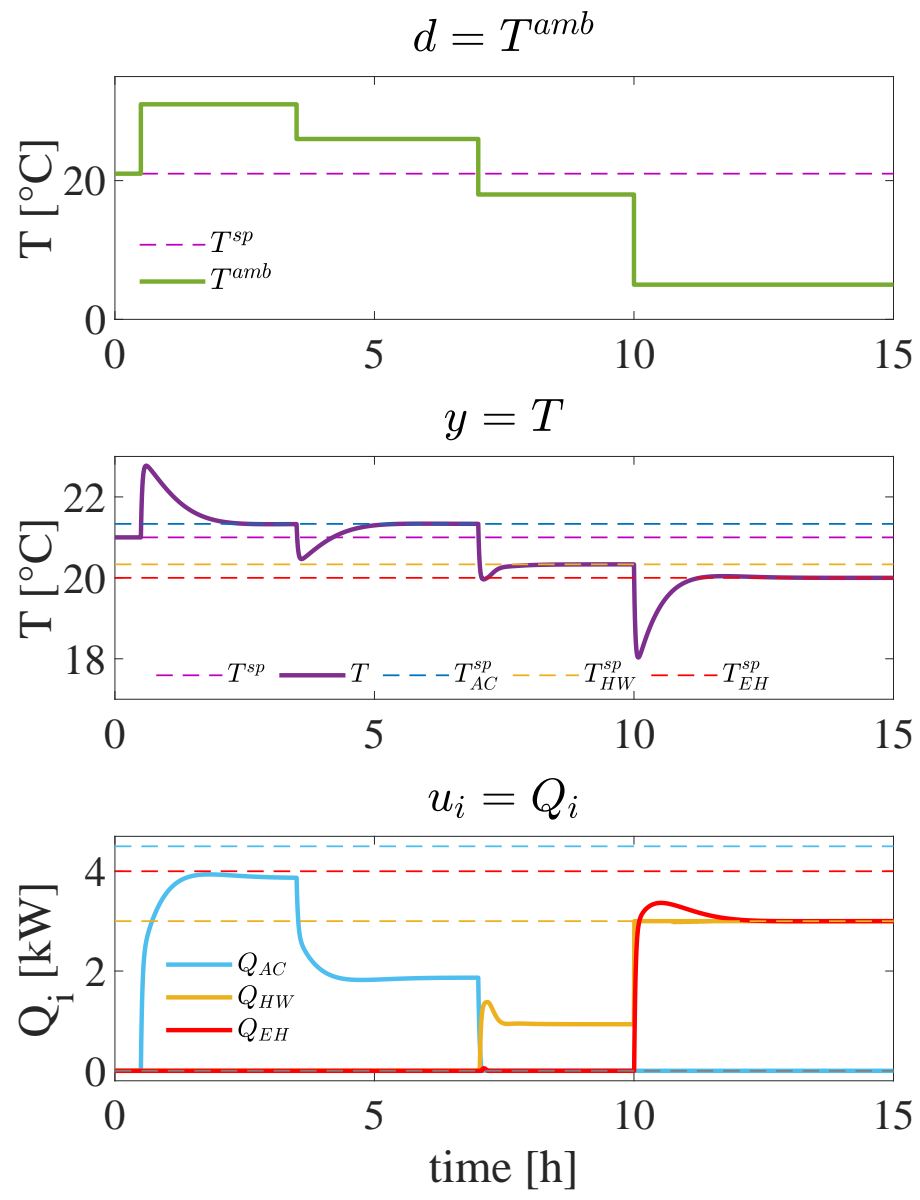

Figure 8. Simulation results using three different controllers, with the optimal setpoint deviations in Table 3. 


\subsection{Comparison with Split Range Control}

We implement a classical split range controller as shown in Figure 9. We use the procedure proposed by Reference [7] to find the tuning parameters for the common controller and the slopes in the split range block (see Appendix C). The common PI-controller has a proportional gain $K_{C}=0.1277 \mathrm{~kW} /{ }^{\circ} \mathrm{C}$ and integral time constant $\tau_{I}=1200 \mathrm{~s}$. For all inputs, the setpoint is always fixed at $y^{s p}=T^{s p}=21^{\circ} \mathrm{C}$, which would correspond to having a huge penalty for setpoint deviation $\left(p_{T} \rightarrow \infty\right.$, in Equation (10)).

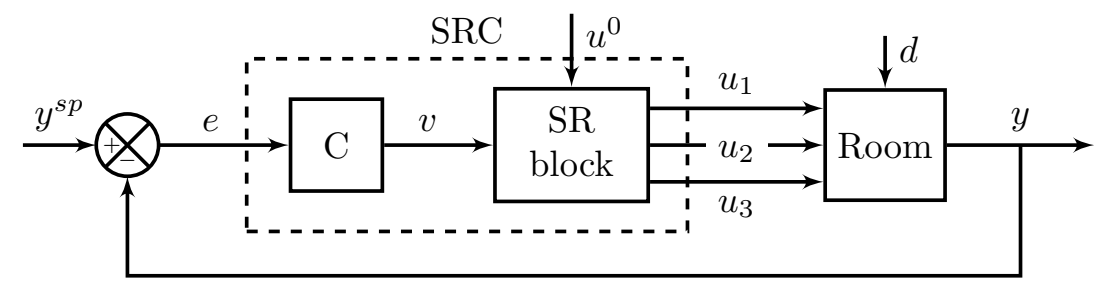

Figure 9. Block diagram for split range control (SRC) with three inputs and one output; $y=T, d=T^{\text {amb }}$ and $u_{i}=Q_{i}$, where $1=\mathrm{AC}, 2=\mathrm{HW}, 3=\mathrm{EH}$. The SR-block is shown in Figure A1.

Figure 10 compares the results of split range control with the previous simulation using three controllers with different setpoints. The changes in $T^{a m b}$ are the same as in Figure 8. We observe that, as expected, that the input (energy) usage is higher with split range control as it has a fixed setpoint.
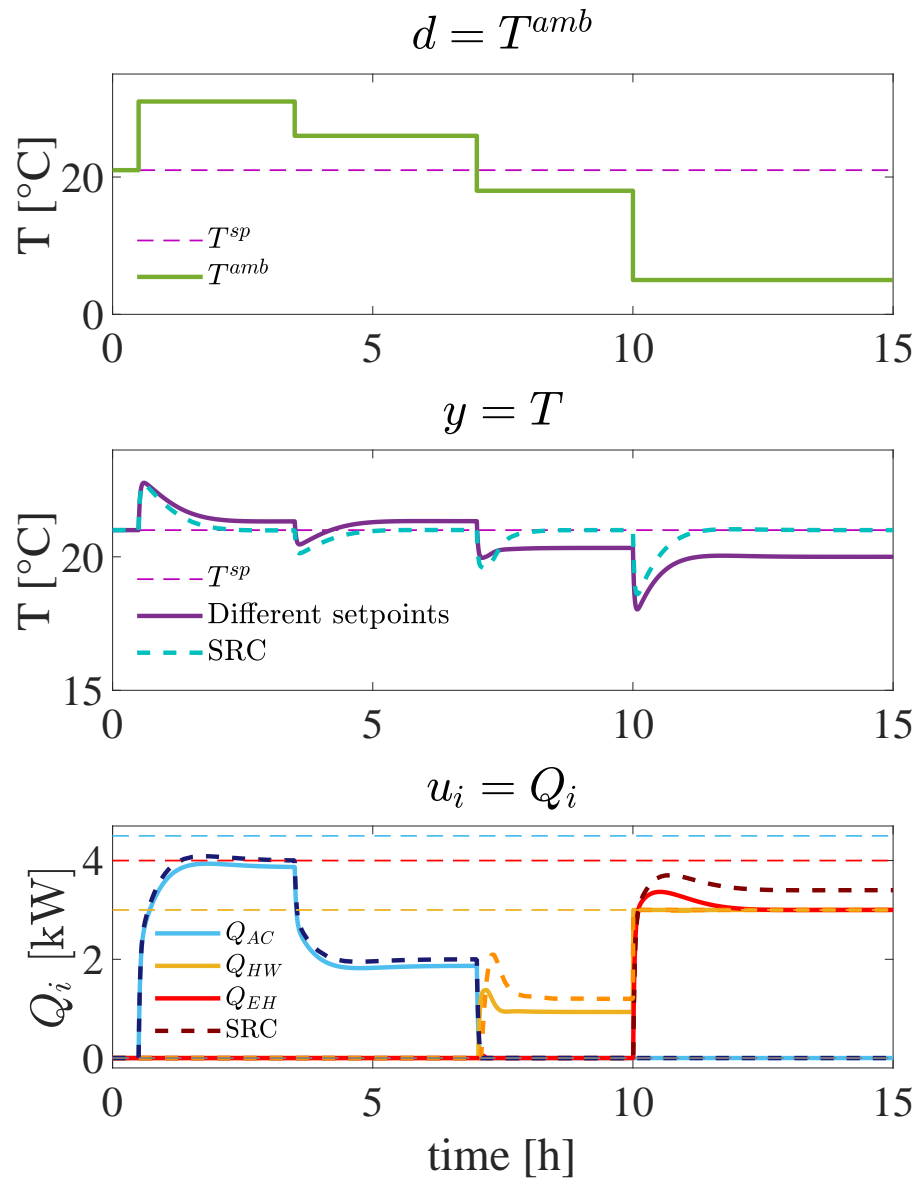

Figure 10. Comparison of simulation results with three controllers with different setpoints (Figure 7) and split range control (SRC, Figure 9). The simulation with three controllers is shown with solid lines and the simulation with SRC is shown with dashed lines. 
Figure 11 shows the accumulated $J_{\text {energy }}$ with both control structures. At the end of the simulation period, energy $_{\text {tot }}=43.15$ [ $\$$ ] with a constant setpoint policy (split range control) and $J^{\text {tot }}=39.84$ [\$] when using optimal setpoints. This corresponds to saving $7.66 \%$ by slightly modifying $T^{s p}$.

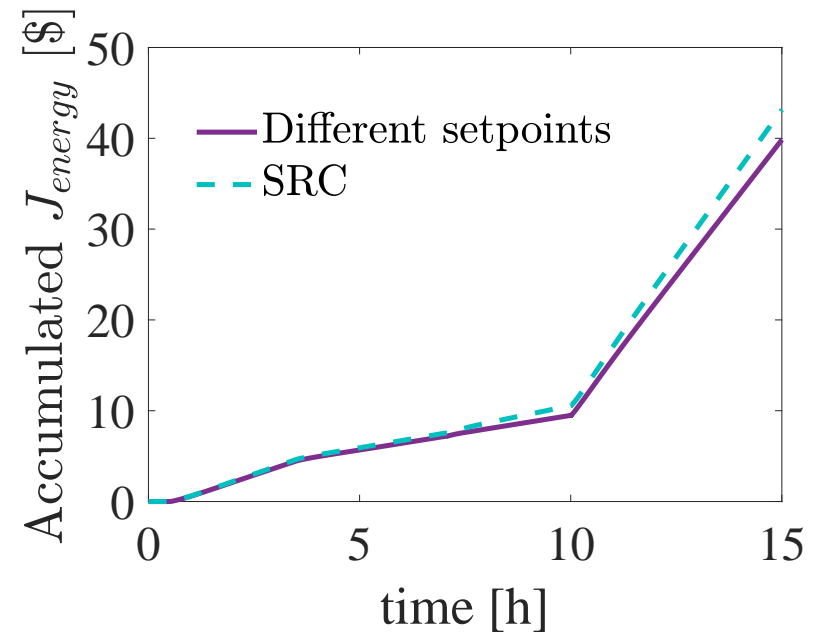

Figure 11. Comparison of accumulated cost of energy $\left(J_{\text {energy }}\right)$ using three controllers with different setpoints (Figure 7) and split range control (SRC, Figure 9).

\section{Discussion}

In this section we first discuss the assumptions made for the cost function. Then we discuss the applicability of the method with different cost functions.

\subsection{Assumptions Made for the Cost Function}

Equations (2) and (10) are steady-state objective functions with a quadratic penalty on the setpoint deviation for the output $\left(\Delta y^{s p}\right)$. This implies that:

- $\quad$ positive and negative deviations are penalized equally

- $\quad$ small deviations are less expensive than large deviations

For inputs, the least expensive steady-state would be to keep them closed $\left(u_{k}=0\right)$. Therefore, all deviations of $u_{k}$ from $u_{k}=0$ have the same sign and we do not need to take any considerations in this respect. We also assume a linear cost in $u_{k}$. Using the example of the case study, this means that the cost of each $\mathrm{kWh}$ is constant for each input, as in Table 2, which is a common case and a reasonable assumption.

\subsection{Applicability of the Method with Different Cost Functions}

The optimal ventilation rate $\left(\dot{m}_{v}\right)$ may be obtained considering outdoor air quality and indoor air quality requirements. Finding appropriate models to define the optimal ventilation rate is an ongoing area or research [11,12]. In our case study, $\dot{m}_{v}$ is constant, considering typical ventilation rates [13]. As it directly affects indoor temperature, it could also be used as an additional input to extend the control range. The usage of $\dot{m}_{v}$ can thus be included in the objective function $J(u, d)$ as the additional term:

$$
p_{v}\left(\dot{m}_{v}^{s p}-\dot{m}_{v}\right)^{2},
$$

where $p_{v}$ is the penalization for deviating from the desired ventilation rate $\left(\dot{m}^{s p}\right)$. The optimal setpoint for room temperature, using $\dot{m}_{v}$ as input, can be found with the procedure described in this work.

With this approach $\dot{m}_{v}$ may be used simultaneously with the rest of the inputs $\left(Q_{i}\right)$. For example, if the ambient air temperature is higher than the temperature inside the room $\left(T^{a m b}>T\right)$, the ventilation should be at its minimum position to introduce only the necessary fresh air and reduce the energy 
consumed by air conditioning [11]. Thus, $\dot{m}_{v}$ and $Q_{A C}$ would be used at the same time and there would always be a deviation from $\dot{m}_{v}^{s p}$.

Alternatively, we can implement a two-step solution. In the first step, we use the procedure illustrated in the case study, only using $Q_{i}$ (cooling or heating) as inputs and keeping the ventilation at its desired setpoint, $\dot{m}_{v}=\dot{m}_{v}^{s p}$. Once that every $\dot{Q}_{i}$ is saturated, we proceed to the second step, in which we use the objective function becomes

$$
J=k_{v}\left(\dot{m}_{v}^{s p}-\dot{m}_{v}\right)^{2}+k_{T}\left(T-T^{s p}\right)^{2} .
$$

Substituting $\dot{m}_{v}$ with the steady-state mass balance, we can derive $T^{\text {opt }}$ and construct a plot similar to that in Figure 6, with $\Delta T=\left(\Delta T^{s p}\right)$ as function of $p_{v} / p_{T}$ and $\dot{m}_{v}^{s p}$.

A third option would be to implement control structure in which we combine input (valve) positioning control with either split range control or multiple controllers. In that case, we could use ventilation as the secondary input $\left(u_{2}\right.$ in Figure 3$)$ to prevent $Q_{A C}$ or $Q_{E H}$ from saturating. In this case, "air quality" requirements may not be always satisfied.

\section{Conclusions}

We proposed a procedure to find optimal setpoints when there is more than one available input for one output. These setpoints can be used to achieve optimal steady-state operation using multiple (PID) controllers, one for each input. The results are valid for problems that can be described with a linear model and in which there is a trade-off between a linear cost for input usage and a quadratic penalty for setpoint deviation.

Using our results, we found optimal setpoints for the control of room temperature using three available inputs. In a simulation case study, we demonstrated that optimal steady-state operation, considering economics and deviation from the desired value, can be reached by using one PI controller for each input, each with a different setpoint. Comparing this implementation with a constant setpoint policy (classical split range control), we obtained a reduction in the energy cost of $7.66 \%$ with only a small setpoint deviation. The benefit of this approach is that optimal steady-state operation can be achieved with negligible computational cost and using PID-control. The ideas discussed in this paper can also be applied to other similar problems and using different types of controllers.

Author Contributions: Conceptualization, A.R.-L. and S.S.; methodology, S.S.; software, A.R.-L.; validation, S.S.; formal analysis, A.R.-L. and S.S.; investigation, A.R.-L.; resources, S.S.; data curation, S.S.; writing-original draft preparation, A.R.-L.; writing—review and editing, S.S.; visualization, A.R.-L.; supervision, S.S.; project administration, A.R.-L.

Funding: This research received no external funding.

Acknowledgments: The authors acknowledge Cristina Zotică for her input.

Conflicts of Interest: The authors declare no conflict of interest.

\section{Abbreviations}

The following abbreviations are used in this manuscript:

$\begin{array}{ll}\text { CV } & \text { Controlled variable } \\ \text { MV } & \text { Manipulated variable } \\ \text { PI } & \text { Proportional-integral } \\ \text { PID } & \text { Proportional-integral-derivative } \\ \text { SRC } & \text { Split range control } \\ \text { VPC } & \text { Valve position control }\end{array}$




\section{Appendix A. Model for Case Study}

The room temperature can be described with the following differential-algebraic system of equations:

$$
\begin{aligned}
m C_{p} \frac{d T}{d t} & =\alpha\left(T^{a m b}-T\right)+\dot{Q} \\
m_{f l} C_{p, f l} \frac{d T_{f l}}{d t} & =\dot{Q}_{H W}-\dot{Q}_{f l} \\
\dot{Q} & =\dot{Q}_{f l}+\dot{Q}_{E H}-\dot{Q}_{A C} \\
\dot{Q}_{f l} & =U_{f l} A_{f l}\left(T_{f l}-T\right) \\
\alpha & =\dot{m}_{v} C_{p}+U_{w} A_{w}
\end{aligned}
$$

The two states in this model are the room temperature $(T)$ and the floor temperature $\left(T_{f l}\right) . T^{a m b}$ is the ambient temperature (outside the room), which is the main disturbance. The main assumptions are: heat losses through the walls $\left(U_{w} A_{w}\left(T^{a m b}-T\right)\right)$, constant ventilation flow which gives a heat loss $\left(\dot{m}_{v} C_{p}\left(T^{a m b}-T\right)\right)$, constant heat capacities $\left(C_{p, i}\right)$, constant air mass inside the room $(m)$ and perfect mixing.

The size of the room is $5 \mathrm{~m} \times 10 \mathrm{~m}$ (floor), with a height of $3.33 \mathrm{~m}$ and with $\mathrm{m} / \dot{m}_{v}=900 \mathrm{~s}=15 \mathrm{~min}$ there are 4 changes of air per hour, which is within requirements for buildings [13]. Table A1 shows the parameters for Equations (A1a)-(A1e).

Table A1. Parameters for room model.

\begin{tabular}{clcl}
\hline Parameter & Description & Value & Units \\
\hline$U_{f l}$ & floor heat transfer coefficient & 10 & $\mathrm{~W} /\left(\mathrm{m}^{2}{ }^{\circ} \mathrm{C}\right)$ \\
$A_{f l}$ & floor area & 50 & $\mathrm{~m}^{2}$ \\
$m_{f l}$ & floor mass & 600 & $\mathrm{~kg}$ \\
$C_{p, f l}$ & floor heat capacity & 1000 & $\mathrm{~J} /\left(\mathrm{kg}{ }^{\circ} \mathrm{C}\right)$ \\
$C_{p}$ & air heat capacity & 1000 & $\mathrm{~J} /\left(\mathrm{kg}{ }^{\circ} \mathrm{C}\right)$ \\
$m$ & mass air in the room & 180 & $\mathrm{~kg}$ \\
$\dot{m}_{v}$ & ventilation flow rate & 0.2 & $\mathrm{~kg} / \mathrm{s}$ \\
$A_{w}$ & wall area & 100 & $\mathrm{~m}^{2}$ \\
$U_{w}$ & wall heat transfer coefficient & 2 & $\mathrm{~W} /\left(\mathrm{m}^{2}{ }^{\circ} \mathrm{C}\right)$ \\
$\alpha$ & Equation (A1e) & 400 & $\mathrm{~W} /{ }^{\circ} \mathrm{C}$ \\
\hline
\end{tabular}

\section{Appendix B. Tuning Parameters for Each Input}

We use the SIMC tuning rules [14] to systematically tune the desired PI controllers for each input. We first identify a first-order plus time delay model for each input $\left(u_{i}\right)$

$$
G_{i}(s)=\frac{K_{p, i}}{\tau_{i} s+1} e^{-\theta_{i} s} \quad \forall i \in\{1,2,3\}
$$

Here, $u_{1}=\dot{Q}_{A C}, u_{2}=\dot{Q}_{H W}$ and $u_{1}=\dot{Q}_{E H}$. Then, we select the desired closed loop time constant $\left(\tau_{c, i}\right)$ to calculate $K_{C, i}$ and $\tau_{I, i}$ :

$$
\begin{aligned}
K_{C, i} & =\frac{\tau_{i}}{K_{p, i}\left(\tau_{c, i}+\theta_{i}\right)} & & \forall i \in\{1,2,3\} \\
\tau_{I, i} & =\min \left\{\tau_{i}, 4\left(\tau_{c, i}+\theta_{i}\right)\right\} & & \forall i \in\{1,2,3\}
\end{aligned}
$$

Skogestad [14] recommends to select $\tau_{c, i}=\theta_{i}$ for tight control; but in many cases, slower tunings $\left(\tau_{c, i}>\theta_{i}\right)$ are used to reduce input usage and improve robustness [15]. 
We use the half-rule $[14,16]$ to approximate the responses to first-order processes with time delay (Equation (A2)). Table A2 shows the parameters of the transfer functions used to find the tuning parameters in Table A3 using Equations (A3a) and (A3b). Due to the floor dynamics, this is a second-order process. We should note that the magnitude of the gain for all inputs is $(\alpha)^{-1}=$ $2.5^{\circ} \mathrm{C} / \mathrm{kW}$. However, the transfer functions for $u_{1}=\dot{Q}_{A C}$ and $u_{3}=\dot{Q}_{E H}$ have positive numerator time constants that need to be approximated. Here we are using Rule T1a in Reference [16] to approximate the transfer function.

Table A2. Parameters for first-order transfer functions for the available inputs.

\begin{tabular}{cccc}
\hline Input & $\boldsymbol{K}_{\boldsymbol{p}, \boldsymbol{i}}\left({ }^{\circ} \mathbf{C} / \mathbf{k W}\right)$ & $\boldsymbol{\tau}_{\mathbf{1}, \boldsymbol{i}}(\mathbf{s})$ & $\boldsymbol{\theta}_{\boldsymbol{i}}(\mathbf{s})$ \\
\hline$u_{1}=\dot{Q}_{A C}$ & -8 & 2968 & 0 \\
$u_{2}=\dot{Q}_{H W}$ & +2.5 & 3058 & 90 \\
$u_{3}=\dot{Q}_{E H}$ & +8 & 2968 & 0 \\
\hline
\end{tabular}

Table A3. PI tuning parameters for the available inputs.

\begin{tabular}{cccc}
\hline Input & $\boldsymbol{\tau}_{c, i}$ (s) & $\boldsymbol{K}_{C, i}$ & $\boldsymbol{\tau}_{\boldsymbol{I}, \boldsymbol{i}}(\mathbf{s})$ \\
\hline$u_{1}=\dot{Q}_{A C}$ & 300 & -1.24 & 1200 \\
$u_{2}=\dot{Q}_{H W}$ & 300 & +3.14 & 1560 \\
$u_{3}=\dot{Q}_{E H}$ & 300 & +1.24 & 1200 \\
\hline
\end{tabular}

\section{Appendix C. Design of the Split Range Controller}

Table A4 summarizes the information that describes the standard split range block for the system analyzed in the case study, Section 4.4. For each input, the SR-block can be represented as the linear function $u_{i}=\alpha_{i} v+u_{i}^{0} \forall i \in\{1,2,3\}$.

The slopes $\left(\alpha_{i}\right)$ in Table A4 are found with the procedure described in Reference [7] using the tuning parameters in Table A3. Here we consider $v=0$ and all inputs closed at the nominal operating point. Note that $\alpha_{i}$ in Table A4 and Figure A1 are the slopes for the split range block described in Section 2 and are not related to $\alpha$ in Equation (A1e) and Table A1.

The bias values in Table A4 are only to be used for the split range controller in Figure 9, with Figure A1 as SR-block. For Figure 7 (different controllers with different setpoints), the bias are the nominal values in Table 1 (all inputs closed).

Table A4. Values for the slopes $\alpha_{i}, \Delta v_{i}$ and $u_{i, 0}$ in the split range block.

\begin{tabular}{crrr}
\hline Parameter & $\boldsymbol{u}_{\boldsymbol{A C}}$ & $\boldsymbol{u}_{\boldsymbol{H}}$ & $\boldsymbol{u}_{\boldsymbol{E} \boldsymbol{H}}$ \\
\hline$\alpha_{i}$ & -9.6829 & 24.5575 & 9.6829 \\
$\Delta v_{i}$ & 0.4647 & 0.1222 & 0.4131 \\
$u_{i}^{0}$ & 0 & 0 & -1.4316 \\
\hline
\end{tabular}

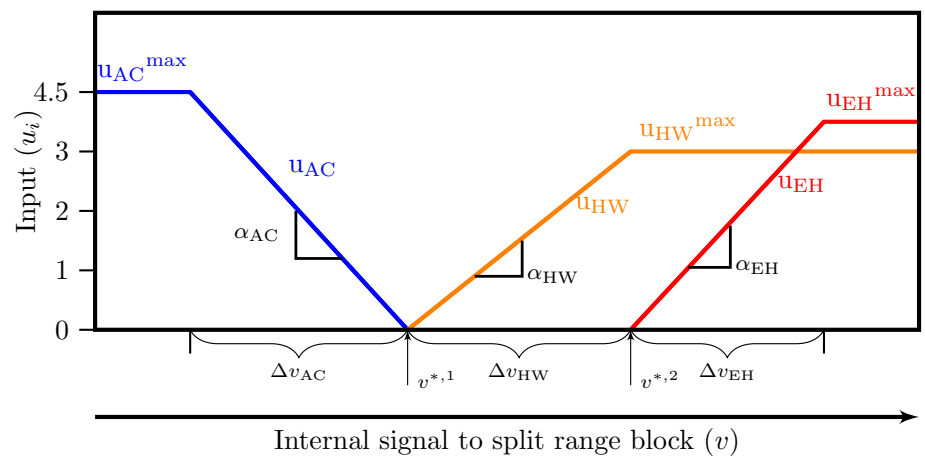

Figure A1. Split range block for controlling room temperature with one source of cooling (AC) and two sources of heating (HW and EH). This is the SR-block in Figure 9. 


\section{References}

1. Eckman, D. Principles of Industrial Control; John Wiley \& Sons: New York, NY, USA, 1945; pp. $204-207$.

2. Fink, E.D. Instruments and Process Control; Delmar Publishers, Inc.: Albany, NY, USA, 1945; pp. 120, 149.

3. Young, A. An Introduction to Process Control System Design; Longmans, Green and Co. Ltd.: London, UK, 1955.

4. Reyes-Lúa, A.; Zotică, C.; Skogestad, S. Optimal Operation with Changing Active Constraint Regions using Classical Advanced Control. In Proceedings of the 10th IFAC Symposium on Advanced Control of Chemical Processes (ADCHEM), Shenyang, China, 25-27 July 2018.

5. Reyes-Lúa, A.; Skogestad, S. Systematic design of active constraint switching using classical advanced control structures. Ind. Eng. Chem. Res. 2019. [CrossRef]

6. Sun, B.X.; Shah, A.; Amalraj, J. A dual split-range control strategy for pressure and flow processes. Control Eng. 2015. Available online: https://www.controleng.com/articles/a-dual-split-range-control-strategy-forpressure-and-flow-processes / (accessed on 9 December 2019).

7. Reyes-Lúa, A.; Zotică, C.; Skogestad, S. Systematic Design of Split Range Controllers. In Proceedings of the 12th IFAC Symposium on Dynamics and Control of Process Systems, including Biosystems, Florianópolis, Brazil, 23-26 April 2019.

8. Shinkskey, F.G. Energy Conservation through Control; Academic Press: New York, NY, USA, 1978; pp. $26-35$.

9. Shinkskey, F.G. Controlling Multivariable Processes; Instrument Society of America: Pittsburgh, PA, USA, 1981.

10. Smith, C.L. Advanced Process Control. Beyond Single Loop Control; John Wiley \& Sons: Hoboken, NJ, USA, 2010; p. 450.

11. Wang, S. Intelligent Buildings and Building Automation, 1st ed.; Spon Press: London, UK, 2010.

12. Ganesh, H.S.; Fritz, H.E.; Edgar, T.F.; Novoselac, A.; Baldea, M. A model-based dynamic optimization strategy for control of indoor air pollutants. Energy Build. 2019, 195, 168-179. [CrossRef]

13. Osborn, P.D. Data charts and tables. In Handbook of Energy Data and Calculations; Elsevier: Amsterdam, The Netherlands, 1985; pp. 1-67. [CrossRef]

14. Skogestad, S. Simple analytic rules for model reduction and PID controller tuning. J. Process. Control 2003, 13, 291-309. [CrossRef]

15. Grimholt, C. Optimal tuning of PID controllers. Ph.D. Thesis, Norwegian University of Science and Technology (NTNU), Trondheim, Norway, December 2018.

16. Skogestad, S.; Grimholt, C. The SIMC Method for Smooth PID Controller Tuning. In PID Control in the Third Millennium; Springer: Berlin, Germany, 2012; pp. 147-175. 